\title{
Semiurgent CABG after Successful PTCA: Should a Patent Stented Coronary Artery Be By-Passed?
}

\author{
Hamdi Tokoz, Soner Sanioglu, Onur Sokullu, Hakan Hasdemir, Azmi Ozler, Serap Aykut Aka, \\ Ibrahim Yekeler, Fuat Bilgen
}

Department of Cardiovascular Surgery, Dr. Siyami Ersek Thoracic and Cardiovascular Surgery Training and Researh Hospital, Istanbul, Turkey.

Email: sanioglu@gmail.com

Received February $21^{\text {st }}, 2012$; revised March $28^{\text {th }}, 2012$; accepted May $4^{\text {th }}, 2012$

\begin{abstract}
Background: After a successful primary percutaneous transluminal coronary angioplasty (PTCA)/stent placement, performing bypass surgery to an artery with a patent stent during semi-urgent coronary artery bypass grafting (CABG) surgeries may protect the patient against ischemic complications due to acute stent occlusion. This issue, however, is controversial. Loss of bypass graft function may occur in the early stages due to the competition between native flow and graft flow. In the present study, the effects of bypass surgery to an artery with a patent stent on the outcomes were investigated. Methods: Fifty nine patients who had undergone isolated CABG surgery within 6 weeks after a successful primary PTCA/stent placement were included in the study. Patients who had undergone emergency operation due to suspicion of acute stent occlusion and the redo cases were excluded in the study. Patients were divided into two groups; those with (Group P, protected) and without (Group NP; non-protected) coronary bypass to an artery with a patent stent. Preoperative, operative and postoperative data were collected retrospectively from the patient files. The need for urgent revascularization was recorded postoperatively. Mann-Whitney $U$ test was used for the analysis of continuous variables, whereas categorical variables were analyzed either using Pearson's chi-square test or Fisher's exact test. Results: Of 59 patients, 35 underwent bypass surgery to the artery with patent stent, whereas bypass was not performed on 24 patients. It was observed that dual antiplatelet therapy had been performed on $20 \%$ of the patients in Group P and $87.5 \%$ of those in Group NP $(p=0.001)$. No acute ischemic change due to stent occlusion was determined in Group P, whereas it was observed in one patient in Group NP $(p=0.4)$. The prevalence of hospital mortality was found to be $8.6 \%$ and $8.3 \%$ in Group P and Group NP, respectively $(p=1)$. Conclusions: Although it was not statistically significant, it was observed that bypass surgery might have a protective effect against stent occlusion in patients who underwent bypass surgery. However, postoperative dual antiplatelet therapy substantially prevented stent occlusion in those without bypass surgery. On the other hand, stent-supported coronary circulation may be unsafe in case of discontinuation of clopidogrel therapy. Therefore, it was suggested that performing bypass surgery to an artery with a patent stent would be more reasonable in patients who underwent semi-urgent CABG surgery.
\end{abstract}

Keywords: Myocardial Infarction; CPI; Stents; Thrombosis; CABG

\section{Introduction}

Primary percutaneous transluminal coronary angioplasty (PTCA) has provided a considerable improvement in the treatment of acute ST-elevation myocardial infarction (STEMI) [1]. It is well known that the use of stent during the procedure enhances the success rate of the process [2]. A certain period of time changing with respect to the stent type is needed for the endothelization of the stent. Until it happens, posponament for elective non-cardiac surgeries is recommended [3]. Because preoperative discontinuation of antiplatelet therapy or postoperative hemodynamic instability may lead to acute occlusion of the stents with incomplete endothelization [4].

In some patients, following a successful primary PTCA/stent placement, coronary artery bypass grafting $(\mathrm{CABG})$ is required before complete endothelization due to critical lesions in the other arteries. Besides the factors related to non-cardiac surgery, cardiac surgery may also lead to acute stent occlusion due to perioperative use of protamine, surgical manipulations causing stent deformation, cardiopulmonary bypass (CPB), or surgery-induced prothrombotic effects [5-7].

In patients who underwent semiurgent CABG after a successful primary PTCA/stent placement, bypassing a patent stented coronary artery may protect the patient 
against acute ischemic complications due to stent occlusion. Although it is controversial, the long-term patency rates between the stent and bypass, which is in favor of bypass, is another reason that supports grafting [8]. Bypass graft performed to an artery with a patent stent may be occluded and loss of function in early period due to native coronary artery flow. In the present study, the effect of bypassing a patent stented coronary artery was investigated by analyzing the outcomes of the patients who underwent semiurgent $\mathrm{CABG}$.

\section{Materials and Methods}

Patients who had admitted to our hospital between January 2004 and September 2010 due to acute STEMI and undergone successful primary PTCA/stent placement using bare metal stents were retrospectively identified. Fifty-nine patients who had undergone isolated CABG surgery before complete endothelization of stent (6 weeks) were included in the study. Indications for CABG in these patients were either critical stenosis in other coronary arteries, or the ischemic symptoms that appeared in the areas apart from the infarct-related artery (IRA) and that could not be improved despite appropriate treatment. Patients who had undergone emergency operation due to acute stent occlusion, as well as the redo cases were excluded in the study.

Patients were divided into two groups; those with (Group P, protected against stent occlusion) and without (Group NP; non-protected against stent occlusion) bypass to a patent stented coronary artery. Demographic data of the patients, such as age, gender, as well as, the presence of hypertension, diabetes mellitus, chronic obstructive pulmonary disease, peripheral artery disease, carotid artery disease, and chronic renal failure, were recorded. Preoperative data of the patients, such as left-ventricle ejection fraction (LVEF), IRA, the timeelapsed between PTCA/stent placement and CABG, use of inotropic support/intraaortic balloon pump (IABP), levels of troponin I (TrI) and creatine kinase (CK)-MB, and use of low-molecular weight heparin (LMWH) and clopidogrel, were determined. Furthermore, operative data, such as the number of bypass grafting, myocardial protection protocols, and the cross-clamp (CC) and CPB times were also determined. Postoperative electrocardiographic (ECG) changes, postoperative concentration of peak serum CK-MB, as well as, TrI, blood urea nitrogen (BUN) and creatinine (Cr) levels, the amount of bleeding within the first 24 hours, the need for reoperation for bleeding, postoperative use of inotropic support/IABP, and postoperative need for urgent revascularization were recorded. Postoperative platelet regimens administered to the patients were recorded. The rates of morbidity and mortality were determined with the leading factors, and all the data between the two groups were analyzed.

\section{Statistical Analysis}

Statistical analysis was performed using SPSS statistical software (version 11.0, SPSS, Inc, Chicago, IL). Continuous variables were expressed as mean \pm standard deviation and compared with Mann-Whitney U test since the sample size was small. Categorical variables were expressed as frequencies and analyzed either using Pearson's chi-square test or Fisher exact test when it was appropriate. A $p$-value $<0.05$ was considered significant.

\section{Results}

Of 59 patients, 35 (Group P) had undergone bypass grafting to a patent stented coronary artery, whereas it was not performed on 24 patients (Group NP). The IRA was the right coronary artery $(\mathrm{RCA})$ in $73 \%(\mathrm{n}=43)$, the circumflex $(\mathrm{Cx})$ artery in $17 \%(\mathrm{n}=10)$, and the left anterior descending artery $(\mathrm{LAD})$ in $10 \%(\mathrm{n}=6)$ of the patients. Saphenous vein graft was used for all coronary arteries except for LAD and left internal mammarian artery was used for LAD. No significant difference was determined between the groups in terms of age, gender, concomitant diseases, and preoperative inotropic support and IABP use. The time-elapsed between the primary PTCA and the surgery was significantly shorter in Group $\mathrm{P}$ as compared to Group NP $(12 \pm 7$ days vs. $18 \pm 9$ days, $p=$ 0.01). There was no significant difference between the groups regarding preoperative BUN, Cr, TrI and CK-MB levels and the left ventricular functions (Table 1).

It was determined that the operation had been performed on beating-heart without using CPB in $5(21 \%)$ patients in Group P and in $3(9 \%)$ patients in Group NP $(p=0.25)$. In patients who were operated with CPB, myocardial protection was provided via antegrade cold blood cardioplegia in $23(72 \%)$ and $16(84 \%)$ of the patients in Group P and Group NP, respectively, whereas it was provided via antegrade/retrograde cold blood cardioplegia in the remaining patients. No significant difference was determined between the groups in terms of myocardial protection protocols.

After the examination of antiplatelet and anticoagulant protocols, it was determined that dual antiplatelet and LMWH therapies had been administered in both groups following the PTCA, clopidogrel had been discontinued 72 hours prior to the procedure, and LMWH and aspirin therapy had been continued. It was observed that postoperative dual antiplatelet therapy had been maintained in $21(87.5 \%)$ and in $7(20 \%)$ patients in Group NP and Group P, respectively $(p=0.001)$. In Group NP, postoperative dual antiplatelet therapy was discontinued due to gastrointestinal bleeding in one patient and hemorrhagic diathesis in two patients.

However, in Group P, it was determined that dual antiplatelet therapy had not been considered as necessary 
Table 1. Preoperative variables of the groups.

\begin{tabular}{|c|c|c|c|}
\hline & Grup $P(n=35)$ & Grup NP $(n=24)$ & $p$-value \\
\hline Age (years) & $60 \pm 10$ & $57 \pm 10$ & 0.20 \\
\hline Male (\%) & $26(74)$ & $23(96)$ & 0.38 \\
\hline HT (\%) & $23(66)$ & $18(75)$ & 0.44 \\
\hline CPOD (\%) & $7(20)$ & $4(17)$ & 1 \\
\hline DM (\%) & $19(54)$ & $16(67)$ & 0.34 \\
\hline PAD (\%) & - & - & - \\
\hline $\mathrm{CKD}^{*}(\%)$ & $2(5.6)$ & - & 0.51 \\
\hline $\operatorname{COD}(\%)$ & $6(17)$ & $4(17)$ & 1 \\
\hline Preop inotrop (\%) & $1(2.8)$ & - & 1 \\
\hline Preop IABP & - & - & - \\
\hline PTCA/CABG int. (days) & $12 \pm 7$ & $18 \pm 9$ & 0.01 \\
\hline Preop BUN (mg/dl) & $19 \pm 8$ & $16 \pm 4$ & 0.31 \\
\hline Preop Cr (mg/dl) & $1.12 \pm 0.7$ & $1 \pm 0.18$ & 0.66 \\
\hline Preop Tr I (ng/ml) & $0.45 \pm 0.49$ & $0.48 \pm 0.76$ & 0.25 \\
\hline Preop CK-MB (U/L) & $16 \pm 8$ & $17 \pm 7$ & 0.79 \\
\hline $\mathrm{LVEF} \leq 35 \%(\%)$ & $4(11.4)$ & $3(12.5)$ & 1 \\
\hline
\end{tabular}

HT: Hypertansion; COPD: Chronic obstructive pulmonary disease; DM: Diabetes Mellitus; PAD: Peripheric artery disease; CKD: Chronic kidney disease; COD: Carotis oclusive disease; IABP: Intra aortic baloon pump; BUN: Blood urea nitrogen; Cr: Creatinine; Tp I: Troponine I; CK-MB: Ceratine kinase-myocardial bant; LVEF: Left ventricle ejection fraction. ${ }^{*} \mathrm{CKD}=$ Glomerular filtratation rate $<60 \mathrm{ml} / \mathrm{min}$.

for the majority of the patients, since grafting to a patent stented coronary artery.

When the operative and postoperative data were compared between the groups, it was found that the number of bypass grafting $(2.97 \pm 0.7$ vs. $2.3 \pm 0.9, p=0.004)$ and the CC time $(60 \pm 18 \mathrm{~min}$ vs. $48 \pm 18 \mathrm{~min}, p=0.01)$ were significantly higher in Group P than Group NP. Moreover, postoperative levels of peak serum CK-MB $(89 \pm 49 \mathrm{u} / \mathrm{L}$ vs. $65 \pm 60 \mathrm{u} / \mathrm{L}, p=0.002)$ and $\operatorname{TrI}(3.01 \pm$ $2.23 \mathrm{ng} / \mathrm{mL}$ vs. $2.16 \pm 5.28 \mathrm{ng} / \mathrm{mL}, p=0.001)$ were also significantly higher in Group P compared to Group NP. Postoperative ECGs of all patients in both groups, except for one in Group NP, revealed no signs of acute myocardial ischemia. On the postoperative 4th hour, the ECG of one patient in Group NP revealed ST-segment elevation in inferior derivations accompanied by hypotension. In this patient, primary PTCA/stent placement was performed on RCA due to acute inferior myocardial ischemia before 11 days, and semi-urgent CABG surgery was planned to be performed due to critical left main coronary artery stenosis. Bypass grafting was not performed to the coronary artery with a patent stent during the operation. However, it was determined that the patient had been re-operated on the postoperative 4th hour due to the above mentioned findings, and saphenous vein bypass grafting had been performed on the RCA. It was observed that the patient had had no problem during postoperative period. No significant difference was determined between the groups regarding other operative and postoperative data (Table 2).

No perioperative mortality was observed among the patients included in the study. The rate of hospital mortality was found to be $8.6 \%(\mathrm{n}=3)$ in Group P and $8.3 \%$ $(\mathrm{n}=2)$ in Group NP $(p=1)$. One of the patients in Group $P$ died of massive pulmonary embolus. It was determined that the other two patients had died due to prolonged mechanical ventilator-associated pneumonia. One of the patients in Group NP weaned from CPB with high-dose inotropic support and died of pump failure on the postoperative 1st day and the other patient had died of uncontrollable upper gastrointestinal bleeding that developed during the postoperative period.

\section{Comment}

Despite the satisfactory studies in the literature concerning acute stent occlusions that develop during non-cardiac surgeries, there are limited numbers of publications about acute stent occlusions that develop during cardiac surgery $[5,6]$. Therefore, the incidence of acute stent occlusion during cardiac surgery is not known 
Table 2. Operative and postoperative variables of the groups.

\begin{tabular}{cccc}
\hline & Group P (n = 35) & Group NP (n= 24) & -value \\
\hline Number of bypass & $2.97 \pm 0.7$ & $2.3 \pm 0.9$ & 0.004 \\
CC time (min) & $60 \pm 18$ & $48 \pm 18$ & 0.01 \\
CPB time (min) & $86 \pm 26$ & $76 \pm 32$ & 0.10 \\
OPCAB (\%) & $3(9)$ & $5(21)$ & 0.25 \\
Postop drainage (ml) & $890 \pm 363$ & $767 \pm 360$ & 0.002 \\
Postop CK-MB (U/L) & $89 \pm 49$ & $65 \pm 60$ & 0.001 \\
Postop Tr I (ng/ml) & $3.01 \pm 2.83$ & $2.16 \pm 5.28$ & 0.35 \\
Postop BUN (mg/dl) & $37 \pm 39$ & $31 \pm 18$ & 0.56 \\
Postop Cr (mg/dl) & $1.7 \pm 1.2$ & $1.4 \pm 0.6$ & 0.39 \\
Postop inotrop (\%) & $14(40)$ & $7(29)$ & 0.63 \\
Postop IABP (\%) & $3(8.6)$ & $1(4.3)$ & $2 \pm 2.1$ \\
ICU duration (days) & $2.7 \pm 5.2$ & $1(4.3)$ & $2(8.3)$ \\
Post-CABGital mortality (\%) & - & $3(8.6)$ & 0.52
\end{tabular}

CC: cross clamp; CPB: Cardiopulmonary bypass; OPCAB: Off-pump coronary artery bypass; CK-MB: Ceratine kinase-myocardial bant; Tp I: Troponine I; BUN: Blood urea nitrogen; Cr: Creatinine; IABP: Intra aortic baloon pump; ICU: Intensive care unit.

exactly. When the postoperative enzyme levels, ECG changes and the rates of mortality and morbidity were examined, it was thought that acute ischemic changes due to stent occlusion had developed in none of the patients in Group P and in one (4\%) patient in Group NP ( $p$ $=0.4$ ). Although no significant difference has been determined between the groups according to the results of the present study, it can be concluded that bypassing a patent stented coronary artery prevents the acute ischemic complications due to stent occlusion. However, this strategy has significantly increased the number of the

bypass grafting, thus the $\mathrm{CC}$ time. In contrast with the expectations, the mean postoperative levels of peak CK-MB and TrI were higher in Group $\mathrm{P}$ as compared to Group NP. These increases did not effect the postoperative clinical course of the patients and it was attributed to the more critical status of the patients and longer $\mathrm{CC}$ time in Group P, rather than acute ischemic events. The time intervals between primary PTCA and CABG were shorter in Group P than in Group NP.

In the present study, the incidence of acute ischemic events due to stent occlusion was found $1.7 \%(n=1)$. Postoperative antiplatelet protocol, as well as the bypass grafting to a patent stented coronary artery, might have played a role in this very low rate. However, adverse effects of this protocol should not be ignored. On the postoperative 4th day, gastrointestinal bleeding developed in one patient in Group NP and this patient died of uncontrolled bleeding. Bypassing a patent stented coronary artery may protect the patient against acute ischemic complications and facilitate bleeding control by means of removing the need for postoperative dual antiplatelet therapy.

It is well-known that postoperative bleeding tendency increases due to the preoperative dual antiplatelet therapy [9]. In the cases with stent-dependent coronary circulation, the discontinuation of clopidogrel therapy due to postoperative bleeding indicates increased risk for acute ischemic complications. Therefore, it may be beneficial to provide stent independent coronary circulation via bypass grafting. In the present study, the rate of emergency resternotomy due to the postoperative bleeding was found to be $10 \%(n=6)$, which was considerably high regarding overall routine $\mathrm{CABG}$ procedures [10]. In Group NP, clopidogrel therapy was discontinued in two patients who were re-operated for bleeding; however, both patients survived without any problem in this risky period. It is obvious that new antiplatelet regimens that would not increase the rate of postoperative bleeding, that would ensure stent patency during preoperative period, and that would be short acting and easily reversible are needed [11].

Bypass graft in an artery with a patent stent may become thrombosed in early period due to native flow competition. In the present study, graft patency was able to be evaluated in only 4 patients who had undergone mediastinal exploration for bleeding. While, 3 bypass grafts were found to be patent, a graft occlusion was ob- 
served. These findings failed to provide adequate information about early patency since there were limited number of patients and the mediastinal explorations had been performed on the day of the operation. The main limitation of the present study was the fact that graft patency could not be evaluated via angiography.

In case of graft occlusion in an artery with a patent stent due to competition, the circulation would again become stent-dependent, and thus clopidogrel therapy would again become important. It was remarkable that acute myocardial ischemia had not been encountered in any of the patients although postoperative clopidogrel had not been used in the majority of the patients in Group P. This was attributed to the facts that either the grafts remained widely patent or the stents which overcame bypass graft flow were resistant against acute occlusion due to their good hemodynamic properties even in the absence of clopidogrel therapy.

\subsection{Limitations of the Study}

There are several limitations of the study because of retrospective design. Evaluation of the early graft patency using cardiac $\mathrm{CT}$ would strengthen our paper but information we have is very limited. However, we have not performed recent patency evaluation because it will demonstrate mid or long-term patency (some of patients was operated six years ago) and this information without early patency rates does not clarify the question which method is better, patent stented coronary artery should be by-passed or not. Occlusion occurred during mid or long-term is multifactorial. Additionally, graft flows and data from autopsies are not available. Because, autopsy and measurement of graft flow are not performed routinely at our institution.

The small number of the patients in the study population is another limitation. But we denote that we need several years to expand the study population. Although our institution is one of the biggest hospitals of Turkey and more than $1000 \mathrm{CABG}$ procedure are performed annualy, the number of patients who can be included study is fifty-nine in the six years interval. Obviously, prospective, multicenteric studies, in which the above mentioned limitation is eliminated, are needed.

\subsection{Conclusion}

In conclusion, bypassing a patent stented coronary artery in semi-urgent $\mathrm{CABG}$ cases leads to elevation in $\mathrm{CC}$ time; however, this elevation makes no significant difference in the clinical course of the patients. Although it was not significant, it was suggested that bypass graft protected the patients from the adverse effects of stent occlusion. In the patients without bypass grafting, stent occlusions were substantially prevented via postoperative dual anti- platelet therapy. However, clopidogrel therapy is likely to be discontinued in any time during postoperative period, and in that case, the stent-dependent coronary circulation might be unsafe. Therefore, we suggest that it is more reasonable to perform bypass to a coronary artery with a patent stent in semi-urgent CABG cases.

\section{REFERENCES}

[1] R. S. Loomba and R. Arora, "ST Elevation Myocardial Infarction Guidelines Today: A Systematic Review Exploring Updated ACC/AHA STEMI Guidelines and Their Applications," American Journal of Therapeutics, Vol. 16, No. 5, 2009, pp. e7-e13.

doi:10.1097/MJT.0b013e31818d40df

[2] M. M. Zhu, A. Feit, H. Chadow, M. Alam, T. Kwan and L.T. Clark, "Primary Stent Implantation Compared with Primary Balloon Angioplasty for Acute Myocardial Infarction: A Meta-Analysis of Randomized Clinical Trials," American Journal of Cardiology, Vol. 88, No. 3, 2001, pp. 297-301. doi:10.1016/S0002-9149(01)01645-9

[3] L. A. Fleisher, J. A. Beckman, K. A. Brown, H. Calkins, E. L. Chaikof, K. E. Fleischmann, W. K. Freeman, J. B. Froehlich, E. K. Kasper, J. R. Kersten, B. Riegel and J. F. Robb, "ACC/AHA 2007 Guidelines on Perioperative Cardiovascular Evaluation and Care for Noncardiac Surgery: Executive Summary: A Report of the American College of Cardiology/American Heart Association Task Force on Practice Guidelines (Writing Committee to Revise the 2002 Guidelines on Perioperative Cardiovascular Evaluation for Noncardiac Surgery)," Circulation, Vol. 116, 2007, pp. 1971-1996. doi:10.1161/CIRCULATIONAHA.107.185700

[4] G. L. Kałuza, J. Joseph, J. R. Lee, M. E. Raizner and A. E. Raizner, "Catasstrophic Outcomes of Noncardiac Surgery Soon after Coronary Stenting," Journal of the American College of Cardiology, Vol. 35, No. 5, 2000, pp. 12881294. doi:10.1016/S0735-1097(00)00521-0

[5] J. M. Alvarez, L. Chen and I. N. Sinclair, "Acute Stent Thrombosis after Off-Pump Coronary Bypass Surgery: A New and Avoidable Complication," The Journal of Thoracic and Cardiovascular Surgery, Vol. 125, No. 6, 2003, pp. 1544-1546. doi:10.1016/S0022-5223(03)00009-6

[6] P. Zurek, M. Gemel, P. Olszówka, W. Domaradzki, D. Szurlej and S. Woś, "In-Stent Thrombosis Following Coronary Artery By-Pass Grafting-A Case Report," Kardiologia Polska, Vol. 59, No. 8, 2003, pp. 142-144.

[7] E. A. Tovar and A. Borsari, "Effects of Surgical Manipulation on Coronary Stents: Should Surgical Strategy Be Altered?" The Annals of Thoracic Surgery, Vol. 63, No. 1, 1997, pp. 37-40. doi:10.1016/S0003-4975(96)00632-7

[8] J. Daemen, E. Boersma, M. Flather, J. Booth, R. Stables, A. Rodriguez, G. Rodriguez-Granillo, W. A. Hueb, P. A. Lemos and P. W. Serruys, "Long-Term Safety and Efficacy of Percutaneous Coronary Intervention with Stenting and Coronary Artery Bypass Surgery for Multivessel Coronary Artery Disease: A Meta-Analysis with 5-Year Patient-Level Data from the ARTS, ERACI-II, MASS-II, and SoS Trials," Circulation, Vol. 118, 2008, pp. 1146- 
1154. doi:10.1161/CIRCULATIONAHA.107.752147

[9] D. Fitchett, J. Eikelboom and S. Fremes, "Dual Antiplatelet Therapy in Patients Requiring Urgent Coronary Artery Bypass Grafting Surgery: A Position Statement of the Canadian Cardiovascular Society," Canadian Journal of Cardiology, Vol. 25, No. 12, 2009, pp. 683-689. doi:10.1016/S0828-282X(09)70527-6

[10] S. Karthik, A. D. Grayson, E. E. McCarron, D. M. Pullan and M. J. Desmond, "Reexploration for Bleeding after Coronary Artery Bypass Surgery: Risk Factors, Outcomes, and the Effect of Time Delay," The Annals of Thoracic Surgery, Vol. 78, No. 2, 2004, pp. 527-534. doi:10.1016/j.athoracsur.2004.02.088

[11] A. M. Pickett, D. A. Taylor and M. Ackman, "Prolonged Infusion of Eptifibatide as Bridge Therapy between Bare-Metal Stent Insertion and Cardiovascular Surgery: Case Report and Review of the Literature," Pharmacotherapy, Vol. 30, No. 4, 2010, pp. 127e-133e. doi:10.1592/phco.30.4.420 